\title{
The Application of Pectin-Insulin Patch on Streptozotocin-Induced Diabetic Rats: Implications in the Hippocampal Function
}

Ntethelelo Sibiya* and Musa Mabandla

Schools of Laboratory Medicine and Medical Sciences, College of Health Sciences, University of KwaZulu-Natal, Durban, South Africa

"Corresponding author: Ntethelelo Sibiya, Schools of Laboratory Medicine and Medical Sciences, College of Health Sciences, University of KwaZulu-Natal, Durban, South Africa; Tel: 27(0)312608602; Fax: 27(0)312607132; E-mail: mpotho@gmail.com

Received date: December 02, 2017; Accepted date: December 13, 2017; Published date: December 23, 2017

Copyright: (C) 2017 Sibiya N, et al. This is an open-access article distributed under the terms of the Creative Commons Attribution License, which permits unrestricted use, distribution, and reproduction in any medium, provided the original author and source are credited.

\section{Abstract}

Background: Diabetics are at high risk of developing dementia associated diseases compared to non-diabetics. The learning and memory impairments manifested in diabetes are attributed to sustained hyperglycaemia. Insulin injections are beneficial in preventing and attenuating the progression of these impairments. However, undesirable effects associated with the current mode of administration remains a challenge. In this study, we evaluated the effects of pectin-insulin patch on learning and memory deficits in diabetic animals.

Methods: Pectin-insulin patches $(20.0,40.8$ and $82.9 \mu \mathrm{g} / \mathrm{kg}$ ) applied on the skin of streptozotocin-induced diabetic rats, thrice daily for 45 days. Learning and memory was assessed using the Morris water maze and the novel object recognition behavioural paradigms. Blood glucose, hippocampal mass, hippocampal insulin receptor, tumour necrosis factor (TNFa) and $\mathrm{C}$ reactive protein (CRP) concentrations were examined.

Results: Patch treatments attenuated diabetes induced hyperglycaemia. The diabetic controls showed significant deficits in learning, spatial and recognition memory. The application of the patch attenuated the learning, spatial and recognition deficits observed in diabetic controls. Diabetic animals presented with up-regulated hippocampal insulin receptor which was not affected by the patch treatment. Patch treatments abolished diabetes induced increases in hippocampal TNF $\alpha$ and CRP concentration.

Conclusions: The pectin-insulin patch improves learning and memory and attenuates hippocampal TNFa and CRP in streptozotocin-induced diabetic rats. These observations may suggest that pectin-insulin patches may present an alternative chronic treatment mode for diabetes considering the challenges associated with insulin injections.

Keywords: Pectin-insulin patch; Glycaemic control; Learning and memory

\section{Introduction}

Clinical studies have shown that a proportion of both type 1 and 2 diabetic patients present with impairment in motor function, problem solving skills and learning and memory [1]. The development of these complications is dependent on the chronicity of the disease and the quality of glycaemic control [2]. While these deficits have been reported to be modest, severe cases have also been reported. Diabetics are at high risk of developing Alzheimer's diseases compared to nondiabetics [3]. In diabetes, a wide spectrum of neuronal alterations has been documented which include synaptic alterations, neuronal degeneration and loss, and cerebral microvascular permeability [4]. These alterations combined together lead to progressive cognitive impairment and increased risk of dementia [5]. Cognitive function is modulated mainly by the hippocampus which is a crucial part of the limbic system [6]. The complexity of the hippocampus makes it one of the most sensitive parts of the brain and is highly susceptible to the metabolic disturbances present in diabetes [7]. In experimental diabetes, the degeneration of hippocampal tissue has been reported and is associated with memory loss [8]. Hyperglycaemia promotes increased production of reactive oxygen species (ROS) which have harmful effects on the central nervous system [9]. Furthermore, an increase in the concentration of inflammatory cytokines such as tumour necrosis factor $\alpha$ (TNF $\alpha$ ) that are associated with diabetes and C-reactive protein (CRP) alter hippocampal synaptic plasticity and have been shown to be neurotoxic [9]. For these reasons, the intense metabolic control particularly by insulin has been shown to be effective in delaying the impairment of cognitive function in diabetes [10]. In the past, it has become apparent that insulin modulate some central nervous system functions [11]. In addition to the presence of metabolic derangements that result from hyperglycaemia, the lack of insulin has been associated with detrimental effects on cognitive function [11]. Insulin receptor signalling is vital for neurotransmitter synthesis and release through the increase in calcium and amino acids uptake [12]. Insulin receptors have been found to be widely expressed in almost all brain regions [13]. Studies have reported that central or peripheral administration of insulin improves learning and memory by interfering with both long term potentiation (LTP) and long term depression (LTD) in part via increased hippocampal plasticity [14]. Furthermore, insulin administration has also been shown to protect the hippocampus against $\beta$ amyloid plaques [15]. Despite the beneficial effects of insulin therapy on improving cognitive function in diabetes, the mode of insulin administration has shortfalls which include anxiety due to multiple injections and episodes of hypoglycaemia [16]. Previously, the pectin-insulin patch which was designed to overcome 
shortfalls of insulin injections was shown to improve metabolic and haemodynamic control in experimental diabetic rats $[17,18]$. In this study, we were interested in the effect of the pectin-insulin patch on learning and memory ability in streptozotocin-induced diabetic rats. Secondly, the study also evaluated the effects on hippocampal insulin receptor expression, TNF $\alpha$ and CRP concentration.

\section{Methods}

\section{Pectin-insulin preparation}

Pectin-insulin patches $(20.0,40.9$ and $82.9 \mu \mathrm{g} / \mathrm{kg})$ were designed using a previously described protocol [18].

\section{Experimental animals}

Male Sprague-Dawley rats (250-300 g) raised in the Biomedical Research Unit of the University of KwaZulu-Natal was used in this study. The animals were kept and sustained under typical laboratory settings (for temperature and humidity) in a $12 \mathrm{~h}$ day: $12 \mathrm{~h}$ night cycle. The animals were permitted contact to water ad libitum and were provided $40 \mathrm{~g}$ standard rat chow daily (Meadow Feeds, Pietermaritzburg, South Africa). All animal studies were revised and accepted by the Animal Research Ethics Committee of the University of KwaZulu-Natal (AREC/080/016D).

\section{Diabetes induction}

Diabetes was developed as formerly defined where rats were given a single intra-peritoneal injection of streptozotocin $(60 \mathrm{mg} / \mathrm{kg})$ freshly prepared in $0.1 \mathrm{M}$ citrate buffer (pH 6.3) [19].

\section{Experimental protocol}

Non-diabetic rats served as the absolute control. STZ-induced experimental animals were divided into 3 groups ( $n=6$ per group). Group 1 was sham treated with pectin patch and served as a negative control. Group 2 was transdermally treated pectin-insulin patch (20.0, 40.9 and $82.9 \mu \mathrm{g} / \mathrm{kg}$, p.o). Group 3 was subcutaneously injected with insulin $(175 \mu \mathrm{g} / \mathrm{kg}, \mathrm{SC})$ and served as the positive control. Two days prior to the application of a pectin-insulin patch, the dorsal region of the rat's neck was smoothly shaven [19]. The pectin-insulin patch was applied three time a day, 8 hours apart for 45 days. At day 35, blood glucose concentration was measured 4 hours post patch application or subcutaneous insulin injection using OneTouch select glucometer (Lifescan, Mosta, Malta, United Kingdom). 24-hour water and food consumption and urine output volume were recorded.

\section{Behavioral tests}

All behavioral tests were performed at 09:00 am. The patches were removed from the animals when performing behavioural tests.

\section{Morris water maze}

The training in the Morris water maze (MWM) was conducted between days 36 and 40 . This paradigm is employed to assess the learning and recall ability of rats and covers exploratory, navigational, spatial and contextual memory [20]. The MWM is made of a $1 \mathrm{~m}$ diameter pool, comprising 4 quadrants. Each quadrant has a cue to help the rat in locating the hidden platform placed in one of the quadrants of the pool. The method consist of placing the rat in a quadrant other than where the hidden platform is located and recording the time taken (escape latency period) by the rat to reach the hidden platform is considered as the animal's ability to learn [20]. The probe test is a post-test for learning which examines the ability of an animal to recall the quadrant in which the hidden platform was located. The time spent in the quadrant with the hidden platform is considered as the ability to remember (memory) [20].

\section{Training and learning}

Animals were exposed to 5 consecutive training sessions for a period of 5 days (day 36-40). Animals were taken to the behavioural room 1 hour prior to the training to allow for familiarisation to the new environment. In the training procedure, each rat was softly placed in the water, head facing the cue in a quadrant. The rat was allowed 120 $s$ to locate the hidden platform. When a rat unsuccessful found the hidden platform in $120 \mathrm{~s}$, it was physically directed by the experimenter on the way to the platform and was allowed $60 \mathrm{~s}$ to explore the platform before being returned to the home cage. Training recommenced the following day for the next 4 days, and time (escape latency period) it took for the rat to locate the platform was recorded. No training took place on day 41 .

\section{Probe test}

On day 42 the probe test was performed. Prior the test, the animals underwent the acclimatisation procedure as previously described. Following removal of the platform from the maze, the animals were allowed $120 \mathrm{~s}$ in the water. Time consumed in the quadrant of the hidden platform was recorded. After the probe test, the animals were returned to the home room.

\section{Novel object recognition test}

The novel objects recognition (NOR) was conducted from day 43 to 45. This task assesses the rodent's capability to recognize a novel object in the environment [21]. The task procedure is comprised of 3 phases: habituation, familiarization and test phase. In the habituation phase (day 43), the animals were taken to the behavioural room 1 hour prior to the test for acclimatisation to the new environment. Afterwards, each animal was permitted to freely explore the open-field arena (40 $\mathrm{cm} \times 40 \mathrm{~cm}$ ) in the absence of objects for $5 \mathrm{~min}$. The rat was then removed from the arena and put back in its holding cage. The following day, during the familiarization phase (day 44), the animals underwent the equivalent acclimatisation procedure as described above. Subsequently, a single rat was placed in the open-field arena containing two undistinguishable sample objects $(\mathrm{A}+\mathrm{A})$ for $5 \mathrm{~min}$. After the familiarisation phase the rats were returned to their home room. After 24 hours (retention time), the test phase (day 45) was performed. In the test phase, the animal was returned to the same open-field arena with two objects, one was identical to the sample and the other one was novel $(\mathrm{A}+\mathrm{B})$ and the rat was allowed to explore for $5 \mathrm{~min}$. During both the familiarization and the test phase, objects were located in conflicting corners. Exploration was considered to have happened when rats confronted the object at a distance of less than 2 $\mathrm{cm}$. Ascending or sitting on the object was not regarded as exploration. Assessment of the episodic memory was considered as a percentage of the recognition index (RI).

$\mathrm{RI}=(\mathrm{N} / \mathrm{N}+\mathrm{F}) \times 100 \%$

$\mathrm{N}=$ time spent exploring the novel object 
$\mathrm{F}=$ time spent exploring the familiar object

\section{Blood and tissue collection}

After experimentation, all the rats were taken to the autopsy room 1 $\mathrm{h}$ before decapitation. The rats were decapitated using a sharp guillotine after-which the trunk blood and hippocampal tissue were collected. Blood was centrifuged for plasma collection at $1000 \mathrm{~g}$. Hippocampal tissue was weighed prior to freezing in liquid nitrogen. All tissue material and plasma were stored in a bio freezer at $-80^{\circ} \mathrm{C}$ until the day of analysis.

\section{Biochemical analysis}

Hippocampal tissue (50 mg) was homogenised in an isolation buffer (0.5 mM Na 2 EDTA, $0.1 \mathrm{M} \mathrm{KH}_{2} \mathrm{PO}_{4}, 0.1 \mathrm{mM}$ dithiothreitol, $0.25 \mathrm{M}$ sucrose) and then centrifuged at $400 \times \mathrm{g}$ for $10 \mathrm{~min}\left(4^{\circ} \mathrm{C}\right)$. The supernatant was harvested and the protein content was quantified using the bicinchininic acid assay (BCA) (Sigma-Aldrich, St Louis, Missouri, USA). Briefly, the standards or samples $(250 \mu \mathrm{l})$ were mixed with BCA working solution $(200 \mu \mathrm{l})$ in a 96 well plate. Thereafter, the plate was incubated at $37^{\circ} \mathrm{C}$ for 30 minutes, after cooling the absorbance was read at $562 \mathrm{~nm}$. The protein concentrations were extrapolated from a protein standard curve $(0.2-1 \mathrm{mg} / \mathrm{mL})$. The supernant was further used for the analysis of insulin receptor, TNF $\alpha$ and CRP expression in the hippocampus.

Plasma insulin and hippocampal insulin receptor, CRP and TNF a concentration were analysed using separate specific ELISA kits (Elabscience and Biotechnology, WuHan) that utilise the SandwichELISA method. The analysis was performed as per manufacturer's instruction. The kits included micro ELISA plates which were coated with antibody specific to insulin, insulin receptor, CRP or TNF a respectively. Standards and samples were pippeted into the appropriate wells of the micro ELISA plate and incubated for 90 minutes. This was followed by the addition of the plate relevant biotinylated detection antibody $(100 \mu \mathrm{l})$. After incubating for 60 minutes, Avidin-Horseradish Peroxidase (HRP) conjugate $(100 \mu \mathrm{l})$ was added to each micro-plate well. After incubating for 30 minutes, the unbound components were washed away. Substrate solution $(100 \mu \mathrm{l})$ was added to each microplate well. After incubating for a further 15 minutes, the stop solution $(50 \mu \mathrm{l})$ was added. The optical density was measured using a Nano spectrophotometer (BMG Labtech, Ortenburg, Baden-Wurttemberg, Germany) at the wavelength of $450 \mathrm{~nm}$. The concentration of the samples was extrapolated from the respective standard curves. For TNF a ELISA kit, detection limit ranged from 31.25-1000 pmol/Ml, intra-assay analytical coefficient of variation ranged from 4.4 to $5.5 \%$ and the inter-assay coefficient variation from 4.7 to $8.9 \%$. The detection limit for CRP ranged from $0.78-25 \mathrm{ng} / \mathrm{mL}$, intra-assay analytical coefficient of variation was $<10 \%$ and the inter-assay coefficient variation from $<10 \%$. The detection limit for CRP ranged from $0.78-25 \mathrm{ng} / \mathrm{mL}$, intra-assay analytical coefficient of variation was $<10 \%$ and the inter-assay coefficient variation from $<10 \%$. The lower and upper limits of detection for insulin receptor were 1.39 and 960 $\mathrm{pmol} / \mathrm{L}$, respectively, the intra-assay analytical coefficient of variation ranged from 4.4 to $5.5 \%$ and the inter-assay coefficient variation from 4.7 to $8.9 \%$.

\section{Statistical analysis}

Data is articulated as means \pm standard error of means (SEM). Statistical analysis was performed using GraphPad Prism Instat
Software (version 5.00, GraphPad Software, San Diego, California, USA). One-way analysis of variance (ANOVA) followed by the TukeyKramer post-hoc test was utilised to analyse the differences among the experimental groups. Values of $\mathrm{p}<0.05$ specify statistical significance.

\section{Results}

\section{Food and water consumption and blood glucose concentration}

Non-diabetic control (ND), diabetic control (DC), patch treatment (20.0, 40.8 and $82.9 \mu \mathrm{g} / \mathrm{kg}$ ) and subcutaneous insulin (SC) groups were analysed for blood glucose concentration, 24-hour food and water consumption at week 5 (Table 1). Hyperglycaemia was present in the diabetic control animals at week 5 (DC vs. ND, p<0.05, Table 1). However, the application of patches $(20.0,40.8$ and $82.9 \mu \mathrm{g} / \mathrm{kg})$ or subcutaneous insulin administration attenuated the blood glucose increase in the diabetic animals (Patch vs. DC and SC vs. DC, $p<0.05$, Table 1). Water and food consumption were elevated in the diabetic controls (DC) in comparison to the non-diabetic (ND) animals (DC vs. ND, $\mathrm{p}<0.05)$. Patch and subcutaneous insulin treatments weakened this increase in food, water intake and urine output volume (Patch vs. DC and SC vs. DC, $\mathrm{p}<0.05)$.

\begin{tabular}{|l|l|l|l|}
\hline $\begin{array}{l}\text { Experimental } \\
\text { groups }\end{array}$ & $\begin{array}{l}\text { Food } \\
\text { consumption } \\
\mathbf{( g / 1 0 0 ~ g ) ~}\end{array}$ & $\begin{array}{l}\text { Water consumption } \\
\text { (ml/100 g) }\end{array}$ & $\begin{array}{l}\text { Blood glucose } \\
\text { (mmol/L) }\end{array}$ \\
\hline ND & $7.5 \pm 0.5$ & $8.40 \pm 1.20$ & $4.9 \pm 0.5$ \\
\hline DC & $21.0 \pm 3.0^{\alpha}$ & $76.46 \pm 7.20^{\alpha}$ & $30.9 \pm 0.6^{\alpha}$ \\
\hline 20 & $11.2 \pm 1.9^{*}$ & $47.25 \pm 4.30^{*}$ & $12.3 \pm 0.8^{*}$ \\
\hline 40.8 & $11.58 \pm 1.9^{*}$ & $41.76 \pm 5.75^{*}$ & $11.2 \pm 0.2^{*}$ \\
\hline 82.9 & $8.9 \pm 1.0^{*}$ & $35.44 \pm 4.23^{*}$ & $9.4 \pm 0.7^{*}$ \\
\hline SC & $8.4 \pm 0.9^{\#}$ & $20.88 \pm 3.20^{\#}$ & $7.4 \pm 0.6^{\#}$ \\
\hline
\end{tabular}

Data is articulated as mean \pm SEM (mean of six samples per group, $n=6$ ) a $=p<0.05$ when comparing $D C$ and $N D{ }^{*}=p<0.05$ when comparing patch and $D C$ and $\#=p<0.05$ when comparing $S C$ and $D C$.

Table 1: Comparison in food, water intake and blood glucose amongst experimental groups at day $35, \mathrm{n}=6$ in each group.

\section{Learning ability}

Learning ability of non-diabetic control (ND), diabetic control (DC), patch treatment (PI) and subcutaneous insulin (SC) groups was analysed using the Morris water maze (Figure 1). Induction of diabetes resulted in a reduced ability to learn as evidenced by a steady reduction in latency period from day 1 to day 5 of training compared to nondiabetic animals a (ND vs. DC, $\mathrm{p}<0.05$, Figure 1 ). Patch treatments and insulin injection resulted in improved learning ability as evidenced by the sharp decrease in latency period from day 1 to day 5 of training $\star$ (DC vs. PI, $\mathrm{p}<0.05$, Figure 1) and \# (DC vs. SC, $\mathrm{p}<0.05$, Figure 1), respectively. 
Citation: Sibiya N, Mabandla M (2017) The Application of Pectin-Insulin Patch on Streptozotocin-Induced Diabetic Rats: Implications in the Hippocampal Function. J Diabetes Metab 8: 779. doi:10.4172/2155-6156.1000779

Page 4 of 8

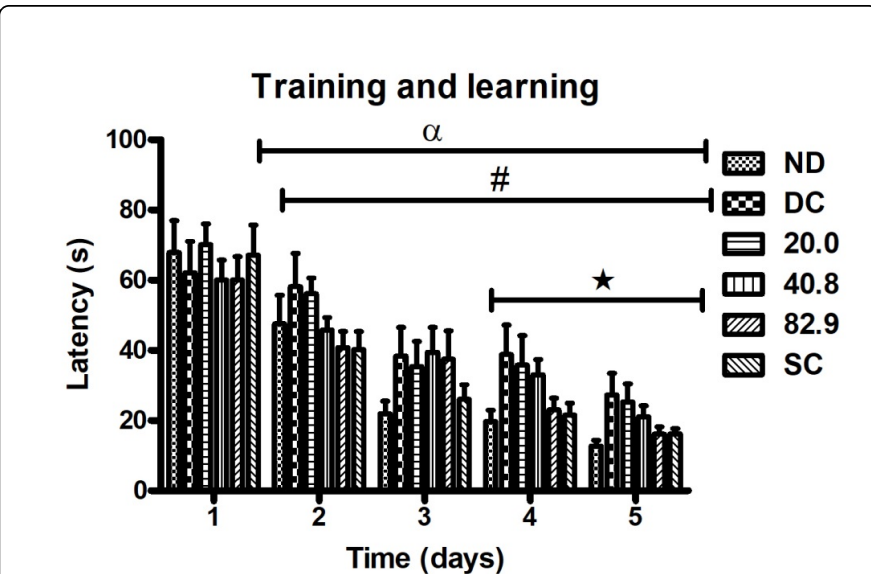

Figure 1: Learning ability in the non-diabetic control (ND), diabetic control (DC), diabetic animals treated with pectin-insulin patches $(20.0,40.8$ and $82.9 \mu \mathrm{g} / \mathrm{kg})$ and diabetic animals subcutaneously administered with insulin (SC). Data is expressed as mean \pm SEM, $\mathrm{n}=6$ in each group. $\alpha=\mathrm{p}<0.05$ when comparing ND and $\mathrm{DC}, \star=$ $\mathrm{p}<0.05$ when comparing DC and $\mathrm{PI}, \#=\mathrm{p}<0.05$ when comparing DC and SC.

\section{Memory retention}

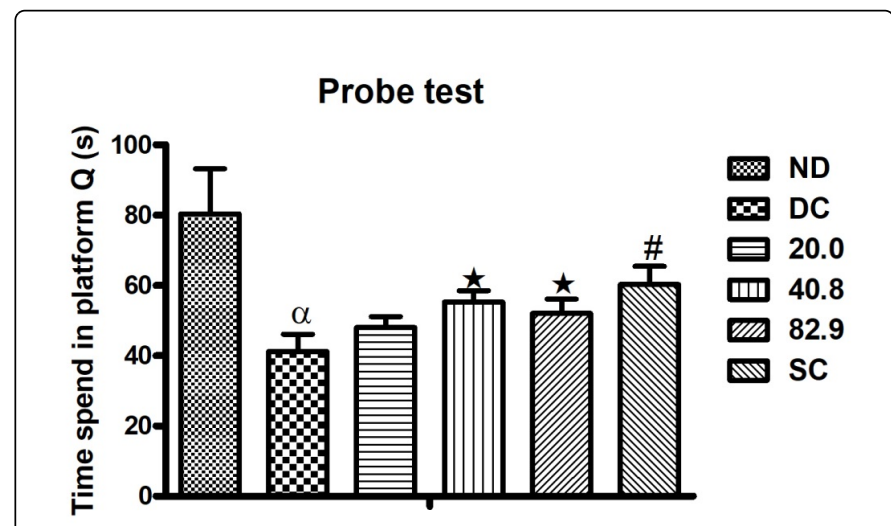

Figure 2: Memory retention in the non-diabetic control (ND), diabetic control (DC), diabetic animals treated with pectin-insulin patches $(20.0,40.8$ and $82.9 \mu \mathrm{g} / \mathrm{kg}$ ) and diabetic animals subcutaneously administered with insulin (SC). Data is expressed as mean $\pm S E M, n=6$ in each group. $\alpha=p<0.05$ when comparing ND and DC, $\star=p<0.05$ when comparing DC and PI, $\#=p<0.05$ when comparing DC and SC.

Memory retention in non-diabetic control (ND), diabetic control (DC), patch treatment (PI) and subcutaneous insulin (SC) groups was analysed using the probe test (Figure 2). Induction of diabetes resulted in a reduced memory recall as evidenced by a decrease in time spent in the platform quadrant $a$ (ND vs. DC, $\mathrm{p}<0.05$, Figure 2). Patch treatments and insulin injection increased time spent in the quadrant with the platform indicating an increase in memory retention $\star$ (DC vs. PI, $\mathrm{p}<0.05$, Figure 2 ) and \# (DC vs. SC, $\mathrm{p}<0.05$, Figure 2 ) respectively.

\section{Recognition memory}

Non-diabetic control (ND), diabetic control (DC), patch treatment (PI) and subcutaneous insulin (SC) groups were assessed for memory recall using the novel recognition test (Figure 3). Induction of diabetes resulted in a reduced memory recall as indicated by a reduction in the recognition index a (ND vs. DC, $p<0.05$, Figure 3 ). Patch treatments and insulin injection resulted in a significant increase in the recognition index $\star$ (DC vs. PI, p $<0.05$, Figure 3 ) and \# (DC vs. SC, $\mathrm{p}<0.05$, Figure 3), respectively.

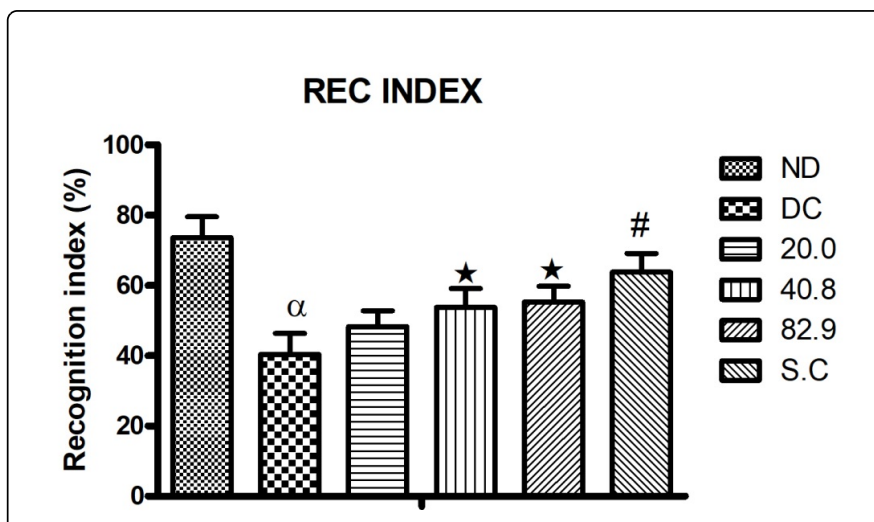

Figure 3: Recognition index (\%) in the non-diabetic control (ND), diabetic control (DC), diabetic animals treated with pectin-insulin patches $(20.0,40.8$ and $82.9 \mu \mathrm{g} / \mathrm{kg}$ ) and diabetic animals subcutaneously administered with insulin (SC). Data is expressed as mean \pm SEM, $n=6$ in each group. $\alpha=\mathrm{p}<0.05$ when comparing DC and ND, $\star=p<0.05$ when PI and DC, \#=p<0.05 when comparing SC and DC.

\section{Plasma insulin}

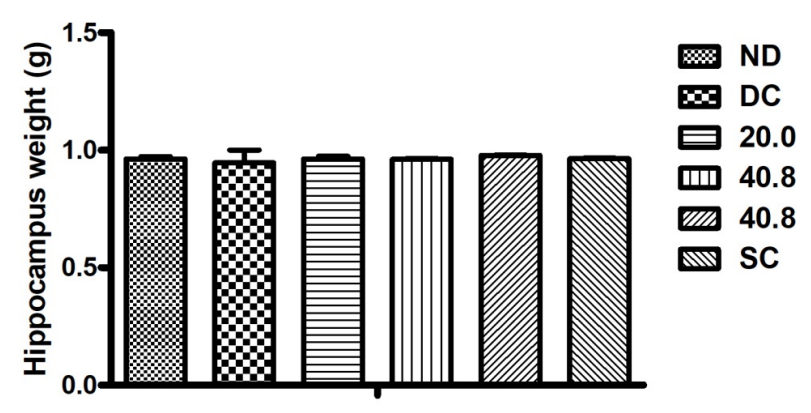

Figure 4: Comparison in hippocampal weights in the non-diabetic control (ND), diabetic control (DC), diabetic animals treated with pectin-insulin patches $(20.0,40.8$ and $82.9 \mu \mathrm{g} / \mathrm{kg}$ ) and diabetic animals subcutaneously administered with insulin (SC). Data is expressed as mean \pm SEM, $n=6$ in each group. 
Non-diabetic control (ND), diabetic control (DC), patch treatment and subcutaneous insulin (SC) groups were analysed for plasma insulin (Figure 4). Induction of diabetes decreased in terminal plasma insulin (ND vs. DC, Table 2). Patch treatments $(82.9 \mu \mathrm{g} / \mathrm{kg})$ and insulin injection resulted in an increase in terminal plasma insulin (Patch treatment vs. DC and SC vs. DC, $\mathrm{p}<0.05$, Figure 4).

\begin{tabular}{|l|l|}
\hline Experimental groups & Plasma insulin $(\mathrm{ng} / \mathrm{mL})$ \\
\hline ND & $9.1 \pm 0.4$ \\
\hline DC & $1.5 \pm 1.3^{\alpha}$ \\
\hline 20 & $1.9 \pm 0.9$ \\
\hline 40.8 & $2.3 \pm 1.0^{*}$ \\
\hline 82.9 & $4.7 \pm 1.3^{*}$ \\
\hline SC & $6.7 \pm 1.2^{\#}$ \\
\hline
\end{tabular}

Data is expressed as mean \pm SEM (mean of six samples per group, $n=6$ ) $\alpha=p<0.05$ when comparing $D C$ and $N D{ }^{*}=p<0.05$ when comparing patch and $\mathrm{DC}$ and \#=p<0.05 when comparing SC and DC.

Table 2: Terminal plasma insulin and blood glucose in the non-diabetic control (ND), diabetic control (DC), diabetic animals treated with pectin-insulin patches $(20.0,40.8$ and $82.9 \mu \mathrm{g} / \mathrm{kg})$ and diabetic animals subcutaneously administered with insulin (SC).

\section{Hippocampal weight}

The hippocampal weights of non-diabetic control (ND), diabetic control (DC), patch treatment (PI) and subcutaneous insulin (SC) groups were compared (Figure 4). Induction of diabetes did not result in a significant change on hippocampal weights. Furthermore, insulin treatments had no significant effect on the hippocampal mass.

\section{Hippocampal insulin receptors}

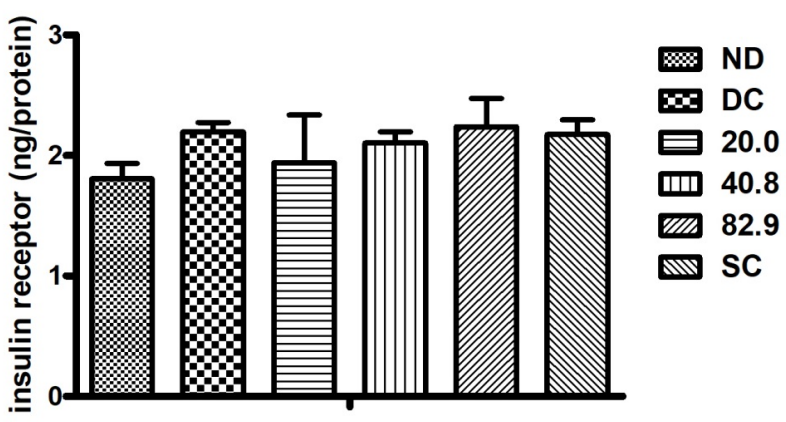

Figure 5: Hippocampal insulin receptor expression in the nondiabetic control (ND), diabetic control (DC), diabetic animals treated with pectin-insulin patches $(20.0,40.8$ and $82.9 \mu \mathrm{g} / \mathrm{kg}$ ) and diabetic animals subcutaneously administered with insulin (SC). Data is expressed as mean \pm SEM, $n=6$ in each group. $\alpha=p<0.05$ when comparing DC and ND.

Hippocampal insulin receptor expression in non-diabetic control (ND), diabetic control (DC), patch treatment (PI) and subcutaneous insulin (SC) groups was analysed (Figure 5). Induction of diabetes resulted in an increase in hippocampal insulin receptor expression $\alpha(\mathrm{ND}$ vs. DC, $p<0.05$, Figure 5). Patch and insulin treatments had no significant effect on hippocampal insulin receptor expression.

\section{Hippocampal TNF $\alpha$ and CRP concentration}

Hippocampal TNFa and CRP in non-diabetic control (ND), diabetic control (DC), patch treatment (PI) and subcutaneous insulin (SC) groups were examined (Table 3 ). The presence of diabetes resulted in an increase in both TNF $\alpha$ and CRP concentration $\alpha$ (ND vs. DC, $\mathrm{p}<0.05$, Table 3 ). The treatment with a transdermal patches or subcutaneous insulin abolished the increase in hippocampal TNFa and CRP concentration $\star$ (DC vs. PI, $\mathrm{p}<0.05$, Table 1$)$ and \#(DC vs. SC, $\mathrm{p}<0.05$, Table 1), respectively.

\begin{tabular}{|l|l|l|}
\hline Experimental groups & TNF $\boldsymbol{\alpha}$ (ng/mg protein) & CRP (pmol/mg protein) \\
\hline ND & $0.1 \pm 0.0$ & $1.0 \pm 0.0$ \\
\hline DC & $0.2 \pm 0.0^{\alpha}$ & $4.1 \pm 0.3^{\alpha}$ \\
\hline 20 & $0.2 \pm 0.0$ & $3.1 \pm 0.1$ \\
\hline 40.8 & $0.1 \pm 0.0^{*}$ & $2.2 \pm 0.2^{*}$ \\
\hline 82.9 & $0.1 \pm 0.0^{*}$ & $1.7 \pm 0.3^{*}$ \\
\hline SC & $0.02 \pm 0.0^{\#}$ & $2.7 \pm 0.2^{\#}$ \\
\hline $\begin{array}{l}\text { Data is stated as mean } \pm \text { SEM (mean of six samples per group) } \alpha=p<0.05 \text { when } \\
\text { comparing DC and ND } *=0.05 \text { when comparing patch and } D C \text { and \#=p<0.05 } \\
\text { when comparing SC and DC. }\end{array}$ \\
\hline
\end{tabular}

Table 3: Comparison in terminal hippocampal TNF a and CRP concentration.

\section{Discussion}

Diabetes is associated with the development and the progression of cognitive deficits including compromised learning and memory [22]. The risk of developing learning and memory deficits is highly dependent of the chronicity as well as the quality control of hyperglycaemia [2]. Adequate glycaemic control has been reported to ameliorate the cognitive disturbances in diabetes [23]. Herein, we are reporting the effects of transdermal application of a pectin insulin patch on learning and memory, hippocampal insulin receptor expression and hippocampal TNF $\alpha$ and CRP concentration in streptozotocin-induced diabetic rats.

The success of diabetes induction was confirmed by the presence of sustained hyperglycaemia. Streptozotocin has been shown to selectively destroy the pancreatic beta-cells mainly via the production of free radicals [23]. As a result, there is a deficit in insulin production which leads to hyperglycaemia [24]. Diabetics require daily insulin administration to achieve glycaemic control [25]. In this study, daily transdermal delivery of insulin attenuated glycaemia in the rats suggesting glycaemic control while augmented plasma insulin concentration. Pectin consists of D-galacturonic acid chains held together by alpha (1-4) glycosidic linkages [26]. In the presence of calcium ions, the adjacent chains of pectin are linked intermolecularly through electrostatic and ionic bonding of carboxyl group with no formation of covalent bonds [27]. At alkaline $\mathrm{pH}$, the low methylated pectin gel has been proven to absorb water resulting in patch distension and this feature perhaps may be a driving force in the 
disintegration of pectin matrix hydrogel which allows the controlled sustained release of the entrapped insulin into the skin thus entering the circulation [27]. Pectin has been employed as a binding agent and allows for a controlled release matrix in tablet formations [28]. The use of dimethyl sulphoxide is envisaged to have aided the permeation of insulin into the circulation perhaps mediated by compromising the integrity of the stratum corneum. Previous studies in our laboratory have demonstrated to offer a sustained controlled release of insulin into the circulation in experimental animals.

Hyperglycaemia is the hallmark of diabetic associated cognitive deficits, therefore its control is critical for positive cognitive function outcomes [29]. In diabetes, a wide spectrum of neuronal alterations has been documented which include synaptic alterations, neuronal degeneration and loss, and cerebral microvascular permeability [30]. In this study, we assessed learning and spatial memory and episodic memory using the Morris water maze and novel object recognition which are behavioural established paradigms. In the present study, the compromised learning, spatial and navigational memory in diabetic rats was observed. Studies conducted by different researchers indicate poor performance of diabetic animals in learning memory experimental paradigms. Biessel et al has demonstrated that there is a compromised place learning together with reduced hippocampal plasticity in diabetic rats [1]. Scientific evidence has also shown that the hippocampus which modulates memory formation is damaged in diabetes [31]. Hyperglycaemia promotes increased oxidative stress which has harmful effects on the central nervous system [32]. Tuzcu et al. has demonstrated that administration of antioxidants such as vitamin $\mathrm{E}$ and quercetin improves memory formation in streptozotocin induced diabetic rats [12]. C-reactive protein (CRP) and diabetes associated inflammatory cytokines such as tumour necrosis factor (TNF $\alpha$ ) alter hippocampal synaptic plasticity and have been shown to be neurotoxic [14]. Furthermore, the a-amino-3hydroxy-5-methyl-4-isoxazolepropionic acid (AMPA) and N-methyl$\mathrm{D}$-aspartate (NMDA) receptors responsible for memory formation are modified and impaired in streptozotocin-induced diabetic rats [33]. In diabetes, due to the increased synthesis and reduced degradation, there is an increase in $\beta$ amyloid accumulation [34]. Furthermore, a diabetic state has been shown impairs hippocampal function through glucocorticoid effects on developing and matured neurons [35]. Recent evidence has suggested that insulin modulates hippocampus metabolic activity and memory formation [36]. Administration of insulin subcutaneously and transdermally improved learning and memory function in diabetic animals. This phenomenon may be attributed to the attenuation of glycaemia which has been shown to have deleterious effects. Secondly, it may also be attributed to the direct effects of insulin in the hippocampus. Insulin has been shown to increase hippocampal plasticity which is critical for long term potentiation thus improving learning and memory [21]. Insulin has also been reported to stimulate the synthesis of AMPA as well as NMDA receptors which are required for memory formation [21]. Reports also indicate that insulin stimulates the entry of calcium ions which are required for the release of excitory neurotransmitters such as glutamate and acetylcholine from the pre-synaptic neurons [37]. Studies on insulin resistant experimental models have also indicated poor cognitive function which may again partly confirm the direct effects of insulin on modulating hippocampal function [38]. The effects of insulin in the hippocampus are mediated through insulin receptor signalling and insulin receptors are highly expressed in the hippocampus in comparison to other regions [39]. Defects in insulin signalling corresponds with the onset and progression of dementia [40]. Insulin receptor regulation in diabetes has been reported to be up-regulated possibly due to the lack of insulin which facilitates receptor downregulation [41]. In this study, the induction of diabetes up-regulated insulin receptors in the hippocampus was mediated perhaps via the increased blood glucose concentration and lack of insulin. However, both insulin administration methods used in this study did not significantly down-regulate hippocampal insulin receptors. The insulin concentration administered might have not had a profound effect on insulin receptor, however, it had resulted in positive learning and memory outcomes perhaps partly via attenuation of hyperglycaemia.

Sustained hyperglycaemia has been shown to cause hippocampal degeneration [11]. Induction of diabetes resulted in a slight decrease in hippocampal size, although not significant it may be of biological significance, especially since memory deficits were observed. The slight increase in the hippocampal size in insulin treated rats may have played a role in maintaining learning and memory function. This feature may also be associated with increased hippocampal plasticity upon insulin administration [21].

In diabetes, the concentration of circulatory inflammatory cytokines such as interleukin 6-18 and TNFa as well as CRP are elevated [42]. The ability of these substances to cross the blood brain barrier causes detrimental effects in the hippocampus since they possess neurotoxic effects [43]. The high levels of hippocampal inflammatory cytokines in diabetic animals may have resulted in memory deficits in this study. Hippocampus has been shown to be sensitive to high CRP concentrations, since high amounts of CRP have been associated with reduced hippocampal mass. Studies report that TNFa has both neuroprotective and neurotoxic effects depending on the receptor subtype [44]. The involvement of TNFa-R 1 is associated with the inhibition of hippocampal plasticity and also promotes apoptosis [14]. Furthermore, TNF $\alpha$ modulates NF- $\kappa \beta$ up-regulation which has been linked to deficits in spatial memory [45]. Inflammatory blockage has been shown to be beneficial through restoration of hippocampal neurogenesis [46]. The attenuation of these inflammatory cytokines as well as CRP in insulin patch treated animals may be attributed to the glucose lowering effects of insulin. Scientific evidence indicates that attenuation of hyperglycaemia has beneficial effects on neutralizing inflammatory cytokine elevation [47].

\section{Conclusion}

The observation in the study suggest that the application of the pectin-insulin patch improve hippocampal function through the attenuation of hyperglycaemia and inflammatory cytokines in diabetes. Furthermore, the therapeutic potency observed is comparable to the traditional insulin injections. In overall, these observations may suggest that the pectin-insulin patch may provide a therapeutic value in the management of diabetes mellitus

\section{Declaration}

We declare no competing interests.

\section{Acknowledgements}

We would like to acknowledge Technology Innovation Agency for funding and the Biomedical Resource Unit personnel for their technical assistance. 


\section{References}

1. Biessels GJ, Gispen WH (2005) The impact of diabetes on cognition: what can be learned from rodent models? Neurobiol Aging 26: 36-41.

2. Watson NA (2015) A randomised trial comparing low-fat diets differing in carbohydrate and protein ratio, combined with regular moderate intensity exercise, on glycaemic control, cardiometabolic risk factors, food cravings, cognitive function and psychological wellbeing in adults with type 2 diabetes: Study protocol. Contemp Clin Trials 45: 217-225.

3. Jolivalt CG (2010) Type 1 diabetes exaggerates features of Alzheimer's disease in APP transgenic mice. Exp Neurol 223: 422-431.

4. Ye L (2011) Diabetes impairs learning performance and affects the mitochondrial function of hippocampal pyramidal neurons. Brain Res 1411: 57-64.

5. Candy SM, Szatkowski MS (2000) Chronic hyperglycaemia increases neuronal sensitivity to high potassium in hippocampal slices from streptozotocin-treated diabetic rats. Neurosci lett 279: 105-108.

6. Klausberger T, Somogyi P (2008) Neuronal diversity and temporal dynamics: the unity of hippocampal circuit operations. Science 321: 53-57.

7. Zlokovic BV (2011) Neurovascular pathways to neurodegeneration in Alzheimer's disease and other disorders. Nature Reviews Neuroscience 12: 723-738.

8. Sima AA, Li ZG (2005) The effect of C-peptide on cognitive dysfunction and hippocampal apoptosis in type 1 diabetic rats. Diabetes 54 1497-1505.

9. Tuzcu M, Baydas G (2006) Effect of melatonin and vitamin E on diabetesinduced learning and memory impairment in rats. Eur J Pharmacol 2006; 537,106-110.

10. Albensi BC, Mattson MP (2000) Evidence for the involvement of TNF and NF-kB in hippocampal synaptic plasticity. Synapse 35: 151-159.

11. Pintana $\mathrm{H}$ (2012) Effects of metformin on learning and memory behaviors and brain mitochondrial functions in high fat diet induced insulin resistant rats. Life Sci 91: 409-414.

12. Moloney AM, Griffin RJ, Timmons S, O'Connor R, Ravid R, et al. (2010) Defects in IGF-1 receptor, insulin receptor and IRS-1/2 in Alzheimer's disease indicate possible resistance to IGF-1 and insulin signalling. Neurobiology of Aging 31: 224-243.

13. Zhao WQ, Chen H, Quon MJ, Alkon DL (2004) Insulin and the insulin receptor in experimental models of learning and memory. Eur J Pharmacol 490: 71-81.

14. Green CJ, Göransson O, Kular GS, Leslie NR, Gray A, et al. (2008) Use of Akt inhibitor and a drug-resistant mutant validates a critical role for protein kinase $\mathrm{B} / \mathrm{Akt}$ in the insulin-dependent regulation of glucose and system A amino acid uptake. J Biol Chem 283: 27653-27667.

15. Haj-Ali V, Mohaddes G, Babri SH (2009) Intracerebroventricular insulin improves spatial learning and memory in male Wistar rats. Behav Neurosci 123: 1309-1317.

16. Moreira PI, Santos MS, Sena C, Seiça R, Oliveira CR (2005) Insulin protects against amyloid $\beta$-peptide toxicity in brain mitochondria of diabetic rats. Neurobiology 18: 628-637.

17. Brunelle RL, Llewelyn J, Anderson JH, Gale EAM, Koivisto VA (1998) Meta-analysis of the effect of insulin lispro on severe hypoglycemia in patients with type 1 diabetes. Diabetes Care 21: 1726-1731.

18. Hadebe S, Ngubane PS, Serumula MR, Musabayane CT (2014) Transdermal Delivery of Insulin by Amidated Pectin hydrogel matrix patch in Streptozotocin-Induced Diabetic Rats: Effects on Some Selected Metabolic parameters. Plos ONE 9: 1-9.

19. Morris R (1984) Developments of a water-maze procedure for studying spatial learning in the rat. J Neurosci Methods 11: 47-60.

20. Hammond RS, Tull LE, Stackman RW (2004) On the delay-dependent involvement of the hippocampus in object recognition memory. Neurobiology of learning and memory 82: 26-34.

21. Broadbent NJ, Gaskin S, Squire LR, Clark RE (2010) Object recognition memory and the rodent hippocampus. Learn Mem 17: 5-11.
22. Gradman TJ, Laws A, Thompson LW, Reaven GM (1993) Verbal Learning and/or Memory Improves with Glycemic Control in Older Subjects with Non-Insulin-Dependent Diabetes Mellitus. J Am Geriatr Soc 41: 1305-1312.

23. Pathan AR, Viswanad B, Sonkusare SK, Ramarao P (2006) Chronic administration of pioglitazone attenuates intracerebroventricular streptozotocin induced-memory impairment in rats. Life sciences 79: 2209-2216.

24. Mythili MD, Vyas R, Akila G, Gunasekaran S (2004) Effect of streptozotocin on the ultrastructure of rat pancreatic islets. Microscopy Research and Technique 63: 274-281.

25. Van den Berghe G, Wouters PJ, Bouillon R, Weekers F, Verwaest C, et al. (2003) Outcome benefit of intensive insulin therapy in the critically ill: insulin dose versus glycemic control. Critical care medicine 31: 359-366.

26. Yapo BM (2011) Pectic substances: From simple pectic polysaccharides to complex pectins-A new hypothetical model. Carbohydrate Polymers 86: 373-385.

27. Axelos MA, Thibault JF (1991) The chemistry of low-methoxyl pectin gelation. . The chemistry and technology of pectin 3: 109-118.

28. Plashchina IG, Braudo EE, Tolstoguzov VB (1998) Circular-dichroism studies of pectin solutions. Carbohyd Res 60: 1-8.

29. Rosenbohm C, Lundt I, Christensen TI, Young NG (2003) Chemically methylated and reduced pectins: preparation, characterisation by $1 \mathrm{H}$ NMR spectroscopy, enzymatic degradation, and gelling properties. Carbohydrate Research 338: 637-649.

30. Krusteva S (1990) Pharmaceutical investigation of a bioerodible nystatin system. Pharmazie 45.

31. Kodl CT, Seaquist ER (2008) Cognitive dysfunction and diabetes mellitus. Endocrine reviews 29: 494-511.

32. Retnakaran R, Zinman B (2008) Type 1 diabetes, hyperglycaemia, and the heart. The lancet 371: 1790-1799.

33. Chilelli N, Burlina S, Lapolla A (2013) AGEs, rather than hyperglycemia, are responsible for microvascular complications in diabetes: a "glycoxidation-centric" point of view. Nutr Metab Cardiovasc Dis 23: 913-919.

34. Biessels GJ, Kamal A, Ramakers GM, Urban IJ, Spruijt BM, et al. (1996) Place learning and hippocampal synaptic plasticity in streptozotocininduced diabetic rats. Diabetes 45: 1259-1266.

35. Anabela P, Palmeira CM (2006) Diabetes and mitochondrial function: Role of hyperglycemia and oxidative stress. Toxicol Appl Pharmacol 212: 167-178.

36. Chabot C, Massicotteac G, Milotb M, Trudeaub F, Gagnéa J (1997) Impaired modulation of AMPA receptors by calcium-dependent processes in streptozotocin-induced diabetic rats. Brain Res 768: 249-256.

37. Takeda S, Sato N, Uchio-Yamada K, Sawada K, Kunieda T, et al. (2010) Diabetes-accelerated memory dysfunction via cerebrovascular inflammation and $A \beta$ deposition in an Alzheimer mouse model with diabetes. Proc Natl Acad Sci USA 107: 7036-7041.

38. Park C (2001) Cognitive effects of insulin in the central nervous system. Neuroscience \& Biobehavioral Reviews 25: 311-323.

39. Messier C (2005) Impact of impaired glucose tolerance and type 2 diabetes on cognitive aging. Neurobiol Aging 26: 26-30.

40. Reagan LP (2007) Insulin signaling effects on memory and mood. Current opinion in pharmacology 7: 633-637.

41. Jolivalt C, Lee CA, Beiswenger KK, Smith JL, Orlov M, et al. (2008) Defective insulin signaling pathway and increased glycogen synthase kinase-3 activity in the brain of diabetic mice: parallels with Alzheimer's disease and correction by insulin. Journal of neuroscience research 86 : 3265-3274.

42. Caro JF, Amatruda JM (1980) Insulin receptors in hepatocytes: postreceptor events mediate down regulation. Science 210: 1029-1031.

43. Tuppo EE, Arias HR (2005) The role of inflammation in Alzheimer's disease. The Int J Biochem Cell Biol 37: 289-305. 
Citation: Sibiya N, Mabandla M (2017) The Application of Pectin-Insulin Patch on Streptozotocin-Induced Diabetic Rats: Implications in the Hippocampal Function. J Diabetes Metab 8: 779. doi:10.4172/2155-6156.1000779

Page 8 of 8

44. Chao CC, Hu S (1994) Tumor necrosis factor-alpha potentiates glutamate neurotoxicity in human fetal brain cell cultures. Dev Neurosci 16: 172-179.

45. Marchetti L, Klein M, Schlett K, Pfizenmaier K, Eisel UL (2004) Tumor necrosis factor (TNF)-mediated neuroprotection against glutamateinduced excitotoxicity is enhanced by $\mathrm{N}$-methyl-d-aspartate receptor activation essential role of a TNF receptor 2-mediated phosphatidylinositol 3-kinase-dependent NF- $\mathrm{B}$ pathway. J Biol Chem 279: 32869-32881.
46. Montgomery SL, Mastrangelo MA, Habib D, Narrow WC, Knowlden SA, et al. (2011) Ablation of TNF-RI/RII expression in Alzheimer's disease mice leads to an unexpected enhancement of pathology: implications for chronic pan-TNF- $\alpha$ suppressive therapeutic strategies in the brain. Am J Pathol 179: 2053-2070.

47. Monje ML, Toda H, Palmer TD (2003) Inflammatory blockade restores adult hippocampal neurogenesis. Science 302: 1760-1765. 